\title{
Apply the systematic risk management-AS/NZS 4360:2004 to operate the project of preoperative evaluation
}

Peng Zhang, Lina Ma, Liquan Wang, Peifen An, Xiaohui Li, Shuangtao Zhao*

Tianjin Hospital, Jiefang South Road, Hexi District, Tianjin, China

Received: September 9, 2020

DOI: $10.5430 /$ jha.v9n5p38
Accepted: November 8, 2020

Online Published: November 13, 2020

\begin{abstract}
Objective: Currently, the medical adverse events stem in part from a lack of significant risk management in preoperative evaluation. This study was to apply the systematic risk management —AS/NZS 4360:2004 to perform the project of preoperative evaluation.

Methods: With the idea of risk management, the doctor's classification and surgery's groups were graded to lay the foundation for project management. Then a preoperative evaluation center was established as a screening role in health management based on AS/NZS 4360:2004.

Results: A total of 144 out of 1,436 patients were identified as ones with much risk mainly including clinical characteristics such as abnormal test $(n=27)$, cardiovascular disease $(n=27)$ and fever $(n=23)$ from pediatric $(35 \%)$, general surgery $(20 \%)$ and trauma $(15.66 \%)$ department. Finally, the potential risk was reduced in the medical process meanwhile the quality of treatment was improved.

Conclusions: This study shows that risk management could be applied into all aspects of hospital management as a drastic and practiced tool.
\end{abstract}

Key Words: Preoperative evaluation, Risk management, AS/NZS 4360:2004, Hospital management

\section{INTRODUCTION}

Preoperative evaluation has eventually improved the quality of care and cost-effectiveness in the hospital, ${ }^{[1,2]}$ which aims to reduce the risks associated with surgery and anesthesia, enhance the quality of perioperative care, restore the patient to the highest level of physical/mental health reasonably possible, and obtain an informed consent for the proposed anesthetic. ${ }^{[3,4]}$ However, more and more admissions for surgical procedures have significantly influenced the evaluation quality before anesthesia despite radical changes in our management. ${ }^{[4]}$ Every quality issue was a risk-management issue and hospitals found ways to integrate risk-management functions. ${ }^{[5,6]}$

As a new systematic discipline, risk management was applied into emergency incident operations and handling the low probability high consequence events, especially in the operations management with environment management. ${ }^{[7,8]}$ As a distinct profession with high technical character and more potential risk, all employees in every position can be exposed to these risks including diagnosis risk, treatment

\footnotetext{
*Correspondence: Shuangtao Zhao; Email: zst-1981@163.com; Address: Tianjin Hospital, No. 406, Jiefang South Road, Hexi District, Tianjin, 300211, China.
} 
risk, technical risk, environmental risk or equipment risk, especially for doctors and nurses. Now, more and more people participated in the medical governance research in the hospital management. However, little attention was paid on the systematic risk management within preoperative evaluation. How to organize a sound medical management mechanism, effectively avoid and transfer preoperative risks, and provide protection for medical staff is an important issue to be solved.

In this study, we developed a Preoperative Evaluation Center (PEC) to conduct a careful preoperative evaluation before surgery to improve patient safety. However, medical adverse events occur frequently due to the lack of significant risk management procedures in preoperative management. Systematic risk management-AS/NZS 4360:2004 was then introduced to carry out preoperative evaluation of all inpatients to improve the quality of care for elective surgery.

\section{MeTHODS}

\subsection{The patient cohort}

A total of 1,436 surgical patients from Tianjin hospital (Tianjin, China) were enrolled into this study. All the patients were diagnosed as orthopedic diseases. And these patients were decided to undergo surgery over the next two days. This research was approved by the institutional review and in line with the principles from the hospital management.

\subsection{Literature review}

To understand the latest development of PEC and through the issue of risk management, we applied the Flinders University Library database "ProQuest Center" to search the literature related to risk management and PEC. This study included the literature on preoperative evaluation of anesthesiologists and risk management of hospital administration.

\subsection{Define the objectives of risk management in PEC}

We summarized all the issues in the medical management and introduced the risk management into the preoperative evaluation management, then identify, analyze, evaluate and control the potential risks based on the plan established previously. The objectives of PEC risk management are as follows: 1) provide guarantee for effective medical services and reduce the occurrence of medical accidents; 2) keep the patient safe and comfortable; 3 ) serve more patients with limited medical resources; 4) maintain the good reputation of the hospital; 5) improve the working environment for all employees.

\subsection{Select the risk management model in PEC}

We developed a risk management model based on the Australian/New Zealand risk management standard AS/NZS 4360:2004 framework. We applied the project management approach to operate the preoperative evaluation process.

Published by Sciedu Press

\subsection{Statistical analysis}

The statistical methods and plots applied into this research were performed with $R$ software (v4.0.2). The statistical significance of differences observed between groups was determined by $\mathrm{t}$ test for the data with normal distribution and non-parametric Kruskal-Wallis test for the one with unnormal distribution when comparing frequencies of clinical factors. All the hypothesis testing in these analyses were performed by a two-sided manner, the statistical significance was defined as $p$-value $<.05$.

\section{RESUltS}

\subsection{Specialists working in PEC}

Most medical errors stemmed primarily from an attenuated risk management in preoperative evaluation. To guarantee the systematic risk management, several professional medical staffs from different disciplines including hospital administration, clinical department and operation room were enrolled into PEC (see Table 1). And the critical value was from the multiple cooperation between PEC and the other administrative departments such as human resource department, nursing department, scientific and educated department and so on. In practice, we first aimed to identify risks and reduce medical adverse events in hospital through the comprehensive work of these experts.

\subsection{Develop the medical team and process of PEC}

We formed a new surgical notice including a comprehensive risk assessment of a patient undergoing surgery in the next day for different diseases (see Figure S1). And it was filled out by the clinician and sent to PEC. Another task for experts in PEC was to divide all doctors into high, medium and low-risk of surgical groups to avoid the critical risk from the operating room (see Figure 1A). And we also developed a sound rules and procedures to smooth the systematic risk management (see Figure 1B). PEC staffs, most clinicians and nearly all patients undergoing elective surgery were optimistic about the project. Such work is necessary to improve the patient safety.

\subsection{Develop principles for preoperative evaluation}

As the population ages, the medical problems of surgical patients became more complex. Therefore, it was very important to establish preoperative evaluation principles after discussion within staffs in PEC (see Table 2). The principle required that all staffs must evaluate operations carefully, especially for ones with much risk. The staff carried on the careful examination and verification to patients receiving different operations in the next day, and proposed the modified suggestions. Eventually, we would report the results to the medical director. 
Table 1. Staffs in PEC

\begin{tabular}{lccll}
\hline Staffs & Gender & Age & Major & Responsibilities \& Functions \\
\hline JW & Male & 54 & Medical superintendor & Director of this center \\
ZG & Male & 56 & Medical management & Be responsible to the medical safety of the project \\
JiW & Male & 62 & Medicine & Evaluate the basic risk of medicine \\
DdL & Male & 61 & Pedo-orthopedics & Evaluate the operative risk of pedo-orthopedics \\
ZgG & Male & 63 & Arthrosis surgery & Evaluate the operative risk of arthrosis operation \\
HyZ & Male & 63 & Spine surgery & Evaluate the operative risk of spine operation \\
PfA & Female & 48 & Nurse & $\begin{array}{l}\text { Secretary, responsible to the operative classification, deploy case data, } \\
\text { feedback the evaluated information to operation room and clinical divisions }\end{array}$ \\
DmX & Male & 59 & Trauma surgery & Evaluate the operative risk of trauma surgery \\
YwS & Male & 58 & Anesthesia & Evaluate the operative risk of anesthesia \\
GrX & Female & 53 & Anesthesia & Evaluate the operative risk of anesthesia \\
StZ & Male & 27 & Blood transplant & Evaluate the risk of transblood in the operation \\
\hline
\end{tabular}

A

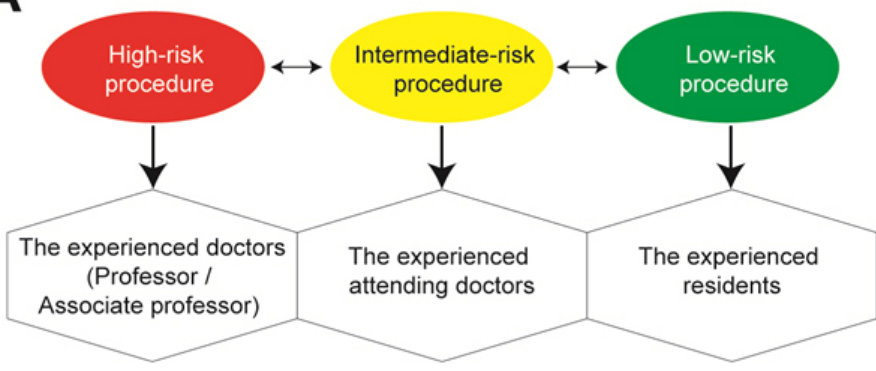

B

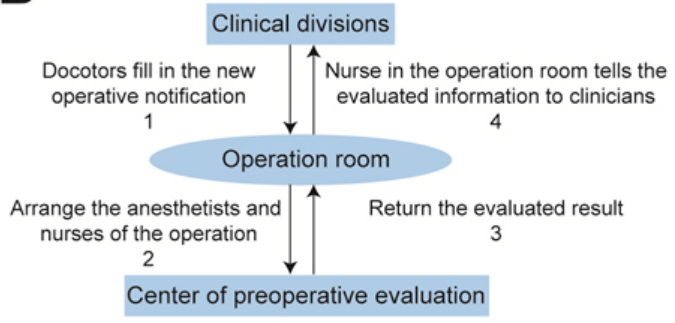

Figure 1. Develop the medical groups and flow-sheet for PEC

A) All clinical operations and doctors were divided into three groups based on the risk management; B) A flow-sheet shows the work steps of PEC

Table 2. The principles of preoperative evaluation

1) We developed the evaluation content based on the major of each staff. The operation would be suspended if there were some serious medical risks ignored by the operative doctors.

2) We audited all the preoperative preparation and would provide some suggestions to the incompleted one.

3) We audited the transplanting blood work in the operation. If the blood type was not enough or rare to meet with the patients, we would give some suggestions.

4) We divided all the operations into three groups: high, intermediate and low-risk, and applied the new operative notification into the groups.

5) We should deal with it carefully for patients with operative risk, and the evaluation should be benefit to the nursing.

6) To improve the medical quality and reduce the risk, the information returned to the clinical divisions must be divided into two groups: the management issues and the medical issues. All the issues must be resolved until the patient meet the standard of operation.

7) Medical superintendor would be responsible for all the process of preoperative evaluation. 


\subsection{Construct the risk management plan of PEC}

Based on the framework of Australian/New Zealand risk management standard AS/NZS 4360:2004, ${ }^{[9,10]}$ we developed a specific preoperative risk management plan for PEC (see Figure 2A). We collected and registered risks in the hospital, and described the risks with the " $3 \mathrm{Cs"}$ " and graded the risks in the medical process. Then the risk quantization matrix was used to show the different risk levels (see Figure 2B). In order to ensure the smooth operation, every employee must take responsibility and perform a great job in each step. Conspicuously, the functions of our risk management plan included monitoring the risk in each link of preoperative evaluation, solving the issues or adverse events encountered in the process of patients' hospitalization, communicating with each other to make sure the patients safe and comfortable or staffs happy in everyday work, even protecting the hospital re- sources and legal rights. As a result, we identified the top 22 risks in PEC and descripted all the consequences, likelihood and acceptable minor risks (see Tables S1-4). Meanwhile, we analyzed the risks in hospital with Root Cause Analysis (RCA) according to the main factors causing adverse events (see Figures S2-3). Finally, the method of qualitative analysis was used to assess the risk results and possibilities, and then the corresponding risk treatment was dealed within each step (see Table S5). Generally, every link of PEC risk management needed continuous monitoring to guarantee the normal operation of internal and external mechanisms. It is clear that we should pay more attention to PEC's risk management plan and take responsibility for all aspects of the process. At the same time, reviewing the status of risk management is a good habit to maintain a risk management system.
A

Risk management process of PEC AS/NZS 4360:2004 - Risk management

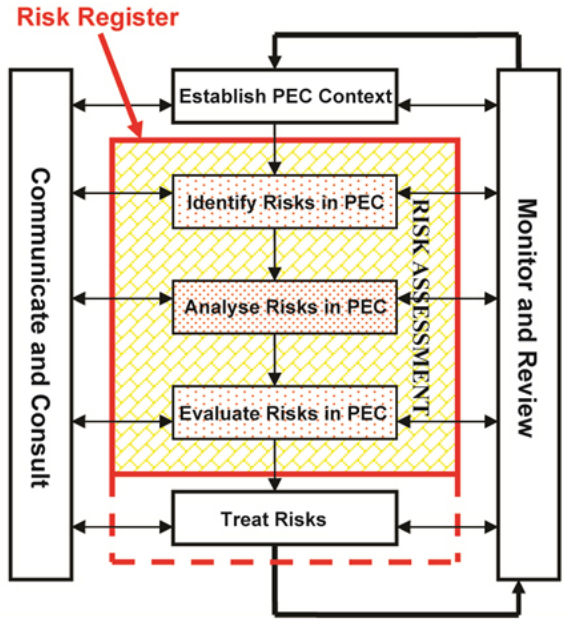

B

\begin{tabular}{|c|c|c|c|c|c|}
\hline \multirow[b]{2}{*}{ Likelihood } & \multicolumn{5}{|l|}{ Consequence } \\
\hline & $\begin{array}{c}\text { Insignificant } \\
1\end{array}$ & $\begin{array}{c}\text { Minor } \\
2\end{array}$ & $\begin{array}{c}\text { Moderate } \\
3\end{array}$ & $\begin{array}{c}\text { Major } \\
4\end{array}$ & $\begin{array}{c}\text { Extreme } \\
5\end{array}$ \\
\hline Almost certain-5 & 5 & 10 & 15 & 20 & 25 \\
\hline Likely-4 & 4 & 8 & 12 & 16 & 20 \\
\hline Possible-3 & 3 & 6 & 9 & 12 & 15 \\
\hline Unlikely-2 & 2 & 4 & 6 & 8 & 10 \\
\hline Remote-1 & 1 & 2 & 3 & 4 & 5 \\
\hline
\end{tabular}

Figure 2. Develop PEC with the risk management standard AS/NZS 4360:2004

A) The plot shows the process of constructing PEC with special risk management; B) The diverse risks grades were displayed with the risk quantification matrix

\subsection{PEC's model played a positive role in risk manage- ment}

After the application of risk management through PEC, medical staff and managers worked closely to monitor and control the medical risks in the preoperative process. Clinical staff performed each operation according to the surgical classification. Of the 1,436 patients, we identified 144 (10\%) patients with high risk and suspended these operations (see Figure $3 \mathrm{~A}$ ). And the top 3 risks in 77 out of 144 patients were from the abnormal test, cardiovascular system risk and fever risk (see Figure 3B). These patients were mainly from the pediatric (35\%), general surgery (20\%) and trauma (15.66\%) department based on the cut-off rate (10\%) of 13 specialties (see Figure 3C). All the results suggested that we should pay more attention to the medical staffs from these three departments, and the administrative department should provide them more training to deal with abnormal test results, cardiovascular disease and fever in order to control the risks in the medical process.

\subsection{Communication and consultation among stakehold- ers were essential in PEC}

In the whole process of risk management, stakeholders in hospital must communicate frequently to solve problems 
in key links. And perfect communication skills will also enhance the cooperation of the whole team in hospital management. Then people could fully understand each other and hospital services were less risky than before (see Figure 4A). Furthermore, consultation played more important role in the risk management process. When regulators encountered conflict and confusion in their work, they would consult each

A

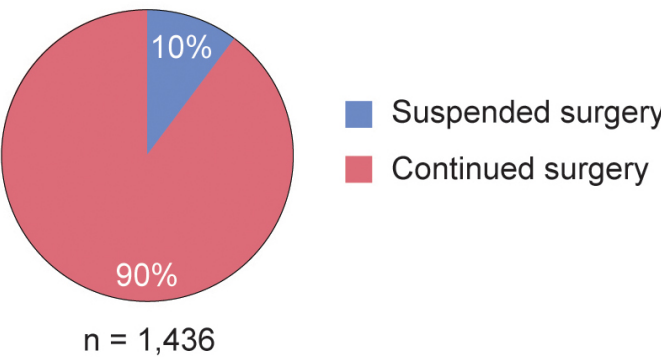

B

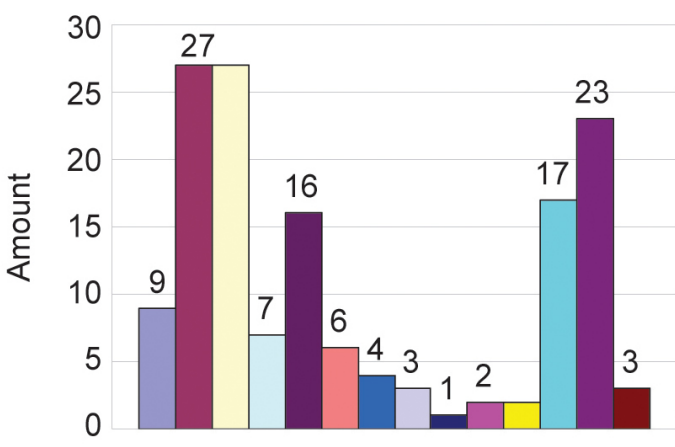

Number of risk for suspending surgery other and conducted the force field analysis ${ }^{[11,12]}$ together (see Figure 4B), and then distinguish between the driving force and the restrain force in the work and develop the most perfect scheme to protect the plan and the hospital reputation. Therefore, this step is necessary to perform the work of risk management well.

C

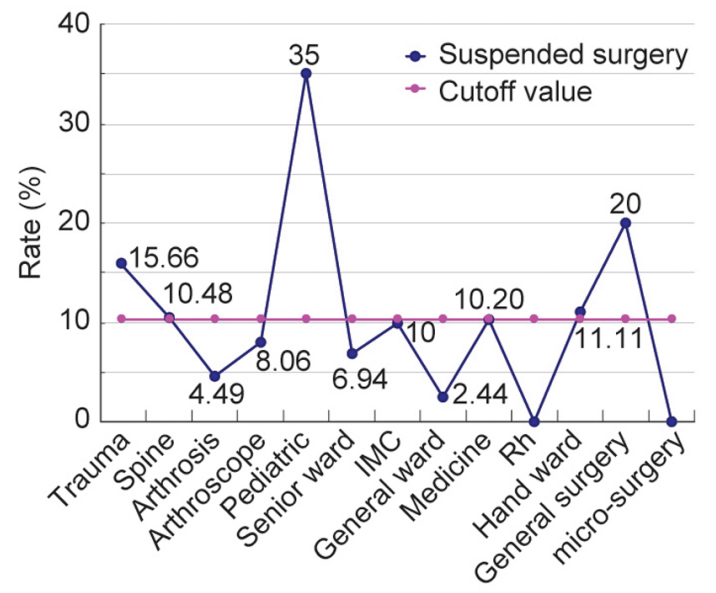

DVT risk

Abnormal laboratory tests risk

Cardiovascular system risk

Respiratory system risk

Fever risk

Metabolism and endocrine system risk

Menses risk of woman

Condition of skin

Drug allergy

Alert the operative schedule

Trouble in machine

Unknown origin

Voluntary suspending operation

Others

Figure 3. PEC reduced the medical hidden dangers and improved the medical quality

A) The pie chart shows the composition of surgery in PEC; B) Medical risks detected from the suspended surgery; $C$ ) The rate of suspended surgery distributed among all the clinical departments

\section{Discussion}

This project was successful in the preoperative management, although there was some conflicts in the process. We organized PEC to improve the preoperative management of the hospital by applying the theory of the systematic risk management. If risk management was used accurately to solve the problems existed in management, it would improve medical quality and doctor-patient relationship and promote the improvement of medical level. Therefore, risk management could deal with complex problems and achieve advanced working efficiency.

There are four factors that affect the success of PEC. Firstly, the success of our program was under the advocating of health care reform. But the medical adverse events occurred frequently due to lack of advantages of preoperative management. That is why we chose to change management. Secondly, we'd better pay close attention to the organizational structure around PEC. We performed force field analysis to 
understand the impact of each organization on the center, and then discovered the key factors corresponding to stakeholders, and took corresponding methods to achieve success based on the characteristics of the group. Thirdly, hospital leaders were involved in the team and provided enough support and reconstruction. At different stages and throughout the life cycle of a project, managers could have the necessary skills to lead a team, and in particular to activate team dynamics, which was a positive factor in a successful. ${ }^{[13,14]}$ A very prominent management style demonstrated that it could improve motivation through participatory leadership style. ${ }^{[15-18]}$ And a project manager could use power or different leadership styles to inspire his team. Fourthly, we valued the art of team management. The best project team was made up of team members closely tied to the goals of the project. They would get personal satisfaction from working on project. ${ }^{[14]}$ In PEC, the staffs with different professions worked hard and efficiently and made up for each other's shortcomings.

A

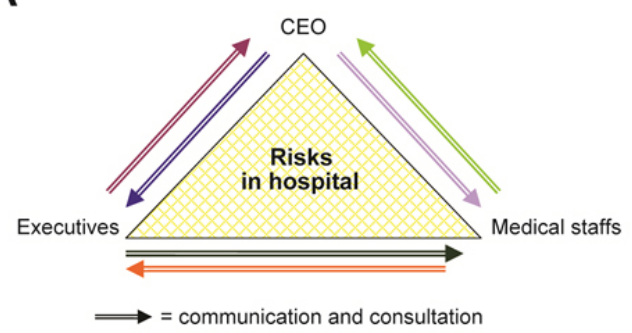

B

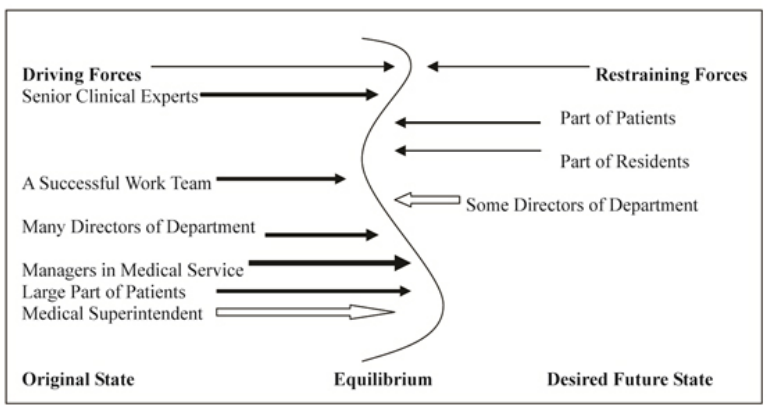

Figure 4. Communication and consultations among all stakeholders

A) Flow sheet for communication in hospital; B) Force field analysis in managing process of PEC

There were some conflicts in the project, although it was successful to apply project management theory into PEC. In this process, conflicts came from various types such as staffs in center, clinicians and patients. Firstly, the conflicts arised from the content of project. At the beginning of the evaluation, 7 clinicians disagreed with hospital administrators on how to complete the project. There was also some debate about the classification of doctors and operations. Secondly, conflicts occurred over the schedule of the project. In the early stage of a project, employees and managers disagreed on when to complete tasks. Thirdly, conflicts occurred in the interpersonal relationship in the project. During the evaluation process, it is inevitable that some patients' surgeries would be suspended due to disqualification. As a consequence, the relationship between the medical staffs in PEC, particularly the doctor-patient relationship, was seriously affected, hindering the development of the project. Some argued that conflicts were everywhere. So, we should try to avoid it. But it was incorrect to try to suppress conflict, because experts had the opportunity to learn some new information to formulate better programs in a convenient way. Through experience, knowledge is gained. ${ }^{[19,20]}$ As part of team construction, project managers and clinicians should recognize that they must develop to resolve conflicts in a project.

As a systematic standard for risk management, AS/NZS4 360:2004 was applied in many areas to deal with actual or potential risk. However, health care is a unique work in society. Because the medical disciplines are developing more slowly than other fields of science, we still have a lot of work to explore the unknown. All of these pose potential risks to medical services. At this time, AS/NZS4360:2004 was introduced into the hospital management. If we would be able to complete the work in accordance with the standard, some risks might be avoided in the process of hospital management.

It was proved that AS/NZS4360:2004 played an important role in our clinical work. In accordance with the core standards of risk management, a special work for preoperative evaluation was organized in hospitals. Through PEC, we have done considerable clinical work to guarantee the surgical safety of inpatients. In the project, we utilized risk identification, risk analysis, risk assessment, risk management and other steps to evaluate the patients' condition before surgery. Then, we communicated with the clinicians, consulted the professors and provided patients with basic treatment to accommodate surgery in the next day. Meanwhile, we would supervise doctors' work in the operating room until the patients achieved the best treatment. And audit was the last step in our implementation of AS/NZS4360:2004 standard, we summarized the risks in clinical work and divided them into several groups, and then develop an implementation plan to solve the issues/ accidents in the corresponding risks.

However, AS/NZS4360:2004 had limited risk prevention and management for special hospitals. Just knowing the core stan- 
dard is not enough, because different enterprises have different culture and connotation. As hospital administrators, we should learn other knowledge to deal with specific problems encountered in practical work. Standards of risk management alone were not sufficient for professional work in health systems. In fact, this is not a weakness that AS/NZS4360:2004 can't overcome, because standards are not fixed and can be developed to adapt to changing environments.

Under the background of medical reform, we usually applied the risk management theory to organize PEC to adapt to the complex medical environment. In practice, risk management made up for the deficiency of preoperative management and played a beneficial role in change management. We took care to avoid recurring problems in the risk management process. Although this standard played an important role in the hospital management, we still need to strive to explore the new orientation to guide our daily work. In other words, Chinese hospitals have their own characteristics, and hospital managers not only make use of professional guidelines, but also create some new ways as typical knowledges.

\section{CONFlicts OF InTEREST Disclosure}

The authors declare they have no conflicts of interest.

\section{REFERENCES}

[1] Allin O, Urman RD, Edwards AF, et al. Using time-driven activitybased costing to demonstrate value in perioperative care: recommendations and review from the society for perioperative assessment and quality improvement (SPAQI). Journal of Medical Systems. 2020; 44: 25. PMid: 31828517. https ://doi.org/10.1007/s10916-019 $-1503-2$

[2] Lemmens L, Van Klei W, Klazinga N, et al. The effect of national guidelines on the implementation of outpatient preoperative evaluation clinics in Dutch hospitals. European Journal of Anaesthesiology. 2006; 23: 962-70. PMid: 16780619. https://doi.org/10.1017/ S0265021506000895

[3] Chuzi S, Wilcox J, Van Wagner LB. The Patient with Preoperative Evaluation. Electrocardiogram in Clinical Medicine. 2020; 239-48. https://doi.org/10.1002/9781118754511.ch24

[4] Garcia-Miguel F, Serrano-Aguilar P, Lopez-Bastida J. Preoperative assessment. The Lancet. 2003; 362: 1749-57. https://doi .org/ 10.1016/S0140-6736(03) 14857-X

[5] Ramos D, Afonso P, Rodrigues MA. Integrated management systems as a key facilitator of occupational health and safety risk management: A case study in a medium sized waste management firm. Journal of Cleaner Production. 2020; 121346. https: //doi.org/10.1016/j.jclepro.2020.121346

[6] Hudson T. Hospitals find ways to integrate risk-management functions. Hospitals. 1992; 66: 32, 4-6.

[7] Cohen MA, Kunreuther H. Operations risk management: overview of Paul Kleindorfer's contributions. Production and Operations Management. 2007; 16: 525-41. https://doi.org/10.1111/j.1937 $-5956.2007 . t b 00278 . x$

[8] Patterson PD, Higgins JS, Van Dongen HP, et al. Evidence-based guidelines for fatigue risk management in emergency medical services. Prehospital Emergency Care. 2018; 22: 89-101. PMid: 29324069. https://doi.org/10.1080/10903127.2017.1376 137

[9] Global S. Australian/New Zealand standard risk management: Tech. Rep; 2004.

[10] Keey R. Risk management: An australasian view. Process Safety and Environmental Protection. 2003; 81: 31-5. https ://doi .org/10 $.1205 / 095758203762851967$
[11] Lawer ET. Examining stakeholder participation and conflicts associated with large scale infrastructure projects: The case of Tema port expansion project, Ghana. Maritime Policy \& Management. 2019; 46: 735-56. https://doi .org/10.1080/03088839.2019.1627 013

[12] Lewin K. Force field analysis. The 1973 annual handbook for group facilitators. 1946; 111-13.

[13] de Melo JCF, Salerno MS, Freitas JS, et al. From open innovation projects to open innovation project management capabilities: A process-based approach. International Journal of Project Management. 2020; 38: 278-90. https://doi.org/10.1016/j.ijprom an. 2020.06 .006

[14] Schmid B, Adams J. Motivation in project management: The project manager's perspective. Project Management Journal. 2008; 39: 60-71. https : //doi .org/10.1002/pmj . 20042

[15] Ellemers N, De Gilder D, Haslam SA. Motivating individuals and groups at work: A social identity perspective on leadership and group performance. Academy of Management Review. 2004; 29: 459-78. https://doi.org/10.5465/amr.2004.13670967

[16] Sirota D, Klein D. The enthusiastic employee: How companies profit by giving workers what they want: FT Press; 2013.

[17] Odoardi C, Battistelli A, Montani F, et al. Affective commitment, participative leadership, and employee innovation: a multilevel investigation. Journal of Work and Organizational Psychology. 2019; 35: 103-13. https : //doi.org/10.5093/jwop2019a12

[18] Koto EA, Samudra AA, Zainal VR, et al. Relationship of work Motivation and participative Leadership with Functional Employment Behavior of Education and Culture of Bengkulu Province, Indonesia. International Journal of Business and Applied Social Science. 2019; 5: 1-19. https ://doi .org/10.33642/ijbass .v5n9p1

[19] Bratianu C, Vătămănescu EM, Anagnoste S, et al. Untangling knowledge fields and knowledge dynamics within the decision-making process. Management Decision. 2020. https ://doi.org/10.110 8/MD-05-2019-0559

[20] Libby R. The role of knowledge and memory in audit judgment. Judgment and Decision-making Research in Accounting and Auditing. 1995; 1: 176-206. https : //doi .org/10.1017/CB09780511 720420.009 